\title{
Bone marrow concentrate injections for the treatment of osteoarthritis: evidence from preclinical findings to the clinical application
}

\author{
Carola Cavallo $^{1} \cdot$ Angelo Boffa $^{2,3}\left(\mathbb{D} \cdot\right.$ Luca Andriolo $^{2} \cdot$ Simone Silva $^{2,3} \cdot$ Brunella Grigolo $^{1} \cdot$ Stefano Zaffagnini ${ }^{2,3}$. \\ Giuseppe Filardo ${ }^{4}$
}

Received: 30 April 2020 / Accepted: 3 July 2020 / Published online: 13 July 2020

(C) The Author(s) 2020

\begin{abstract}
Purpose To investigate the available literature on the use of bone marrow aspirate concentrate (BMAC) and summarize the current evidence supporting its potential for the injective treatment of joints affected by osteoarthritis (OA).

Methods A systematic literature search was conducted on three electronic databases (PubMed, Embase, and Cochrane Library) in April 2020, using the following string: "((bone marrow concentrate) OR (BMC) OR (bone marrow aspirate concentrate) OR (BMAC)) AND (osteoarthritis)", and inclusion criteria: clinical and preclinical (animal) studies of any level of evidence, written in English language, and evaluating the intra-articular or subchondral use of BMAC for the injective treatment of OA joints.

Results The publication trend remarkably increased over time. A total of 22 studies were included in the qualitative data synthesis: four preclinical studies and 18 clinical studies, for a total number of 4626 patients. Safety was documented by all studies, with a low number of adverse events. An overall improvement in pain and function was documented in most of the studies, but the clinical studies present significant heterogeneity, few patients, short-term follow-up, and overall poor methodology.

Conclusion There is a growing interest in the field of BMAC injections for the treatment of OA, with promising results in preclinical and clinical studies in terms of safety and effectiveness. Nevertheless, the current knowledge is still preliminary. Preclinical research is still needed to optimize BMAC use, as well as high-level large controlled trials to better understand the real potential of BMAC injections for the treatment of patients affected by OA.
\end{abstract}

Keywords Bone marrow concentrate $\cdot \mathrm{BMAC} \cdot$ Injective $\cdot$ Intra-articular $\cdot$ Osteoarthritis $\cdot$ Cartilage

\section{Introduction}

Osteoarthritis (OA) is a degenerative disease characterized by progressive deterioration and loss of articular cartilage with concomitant structural and functional changes in the entire joint [1].

Angelo Boffa

angeloboffa@libero.it

1 Laboratorio RAMSES, IRCCS Istituto Ortopedico Rizzoli, Via di Barbiano, 1/10, 40136 Bologna, Italy

2 Clinica Ortopedica e Traumatologica II, IRCCS Istituto Ortopedico Rizzoli, Via Pupilli, 1, 40136 Bologna, Italy

3 Alma Mater Studiorum - Università di Bologna, Via Zamboni, 33, 40126 Bologna, Italy

4 Applied and Translational Research (ATR) Center, IRCCS Istituto Ortopedico Rizzoli, Via di Barbiano, 1/10, Bologna, Italy
Its incidence and prevalence are rising, in particular among older adults in developed countries, likely related to the aging of the population and increasing obesity [2-4]. Clinical features of OA are mostly characterized by signs and symptoms of inflammation, including pain, effusions, stiffness, and loss of mobility, often associated with significant functional impairment [5]. Current management strategies include weight loss, physical treatments, oral medications, such as non-steroidal anti-inflammatory drugs and acetaminophen, and intra-articular injection therapies with corticosteroids and hyaluronic acid (HA) [6]. Nevertheless, these treatment options mainly provide symptom relief rather than disease-modifying changes to the intra-articular environment [7].

The use of orthobiologics is gaining increasing interest due to the availability of new promising products to address OA. Among these, platelet concentrates are now considered a suitable option to treat $\mathrm{OA}$, with results going beyond the mere placebo effects expected for injective treatments and higher than those provided by other traditional products, although 
with still suboptimal outcomes $[8,9]$. More recently, mesenchymal stromal cells (MSCs) have been proposed as a promising alternative for OA treatment thanks to their structural contribution to tissue repair and even more their immunomodulatory and anti-inflammatory actions, through direct cell-tocell interaction or secretion of bioactive factors $[10,11]$. MSCs can be easily isolated from various tissues, such as bone marrow, adipose tissue, synovial membrane, peripheral blood, and skin [12]. Bone marrow MSCs (BMSCs) were the first type of MSCs to be identified and, due to the ease of collection, bone marrow currently represents a commonly used source of MSCs [13]. In particular, this type of MSCs has been either applied as a cell suspension after being expanded by culture or used as a simple bone marrow concentrate (BMC), thanks to their relative abundance [14]. Considering the strict regulations and the problems related to cell manipulation and expansion, cultured BMSCs have been widely explored in the preclinical setting, but their use is extremely limited in clinical practice, both in Europe and in the USA [15-19]. Conversely, the minimal cell manipulation approach, allowing to obtain a bone marrow aspirate concentrate (BMAC) directly on site in a one-step treatment, has been widely utilized in the clinical practice for the treatment of cartilage lesions first and, more recently, has been proposed as a promising injective approach to treat degenerative orthopaedic conditions $[20,21]$. Nevertheless, the real potential of BMAC as intra-articular OA treatment remains controversial [22].

The aim of this systematic review was to examine the available literature on the use of BMAC, evaluating preclinical and clinical studies, in order to summarize the current evidence supporting its potential for the injective treatment of joints affected by OA. The hypothesis of the current study was that the available preclinical studies support the rationale for the injective use of BMAC, and that an increasing number of clinical studies reports safety and effectiveness of this biological approach.

\section{Material and methods}

A systematic review of the literature was performed on the use of BMAC as injective treatment for joints affected by OA. A literature search was conducted on three electronic databases (PubMed, Embase, and Cochrane Library) on April 20th, 2020, with no time limitation and without any filters, using the following string: "((bone marrow concentrate) OR (BMC) OR (bone marrow aspirate concentrate) OR (BMAC)) AND (osteoarthritis)". According to the Preferred Reporting Items for Systematic Reviews and Meta-Analysis (PRISMA) and Cochrane guidelines [23], the article selection and data extraction process were conducted separately by two authors $(\mathrm{AB}$ and SS). The initial title and abstract screenings were made using the following inclusion criteria: clinical and preclinical (animal) studies of any level of evidence, written in English language, and evaluating the intra-articular or subchondral use of BMAC for the injective treatment of joints with OA. Exclusion criteria consisted of articles written in other languages, literature reviews, basic science in vitro articles, case reports, congress abstracts, studies on joint diseases different from OA, studies on other BMAC applications (e.g., use as augmentation to other surgical techniques), and studies dealing only with expanded or otherwise manipulated MSCs. In the second step, the full texts of the selected articles were screened, with further exclusion according to the previously described criteria. Additionally, all references from the selected papers and previously published relevant reviews were also screened. Two investigators independently reviewed each article (AB and SS), and any discrepancies between them were resolved by discussion and consensus. For the included studies, relevant data were extracted from article texts, tables, and figures, and then summarized and analyzed according to the purpose of the present work. In particular, the following data were collected for preclinical studies: year of publication, animals evaluated, joint involved, OA model, types of treatment, BMAC harvest site and characteristics, follow-up length, evaluation methods, and results. For clinical studies, the following data were collected: year of publication, study design, joint involved, treatment type and schedule, BMAC manufacturing and characteristics, number of evaluated subjects, subject's characteristics, follow-up length, main results, and adverse events.

The effectiveness of BMAC injective therapy was evaluated by summarizing the reported benefits, while the safety of the procedures was evaluated identifying the reported side effects. To assess the methodological quality of the included clinical studies, the subscales of a Coleman Methodology Score, modified by Kon et al. [24], were determined for each study by two separate authors (AB and SS). In case of disagreement between the two authors, divergences were discussed and a consensus was reached.

\section{Results}

The flowchart of the article selection process is reported in Fig. 1. After duplicates were removed, the initial search identified 2027 records, whose abstracts were screened and selected according to the inclusion/exclusion criteria. Four articles were identified through the reference lists, which gave a total of 29 articles assessed for eligibility. However, five studies were excluded being congress abstracts, one study was excluded because it was a case report [25], and one study was excluded because it provided the same data of another included study [26]. Thus, a total of 22 studies were included in the qualitative data synthesis: 4 preclinical studies [27-30] and 18 clinical studies [31-48]. Since the first reports in 2014, the 
Fig. 1 Flowchart showing the systematic review selection process

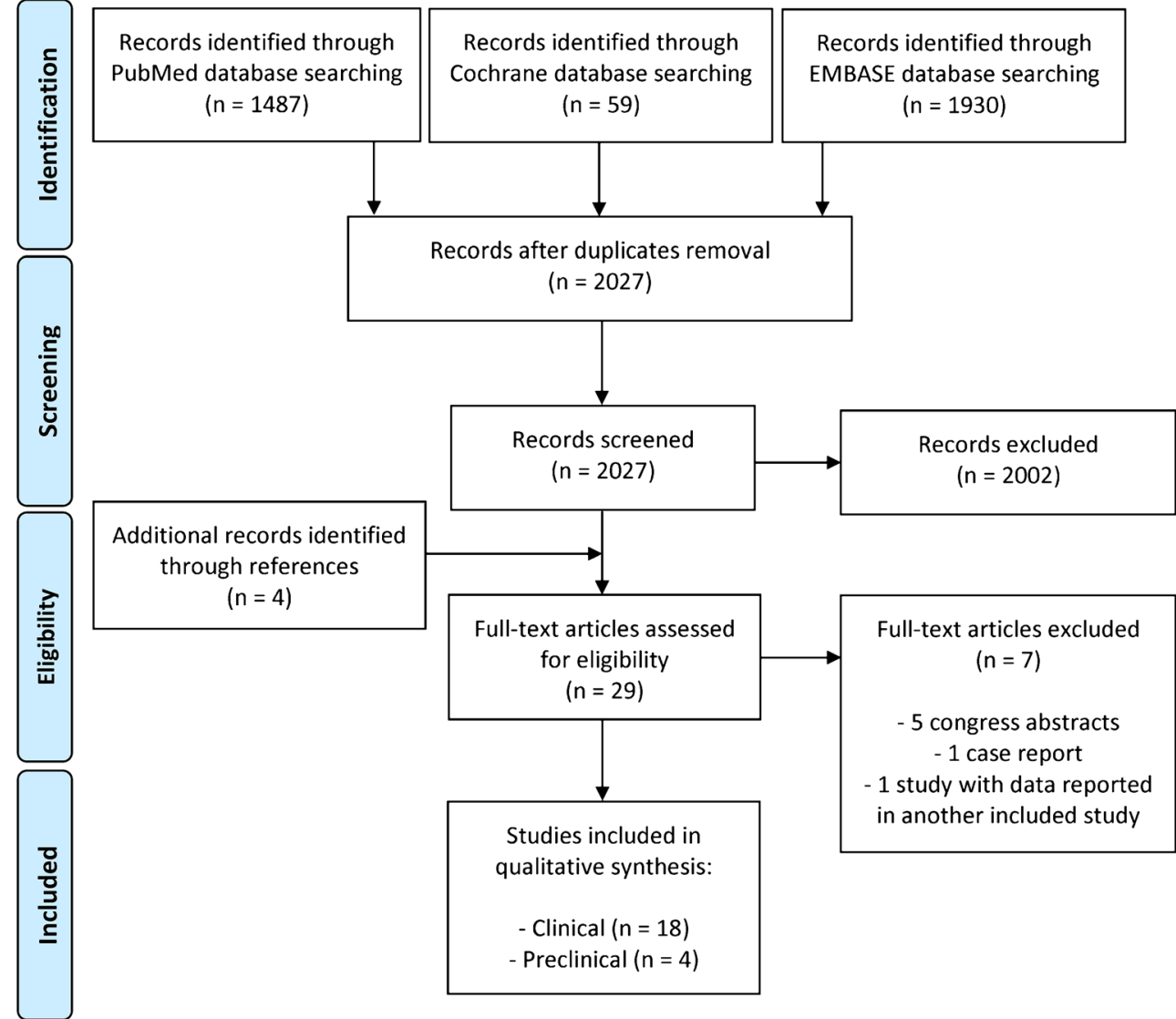

publication trend remarkably increased over time, especially for the clinical studies, with over $50 \%$ of the articles published from 2018 (Fig. 2).

\section{Preclinical studies}

The potential of BMAC for the injective OA treatment has been tested in 4 animal studies (Table 1). All of them were comparative studies investigating the results of BMAC in knee OA models against placebo (saline), HA, platelet-rich plasma

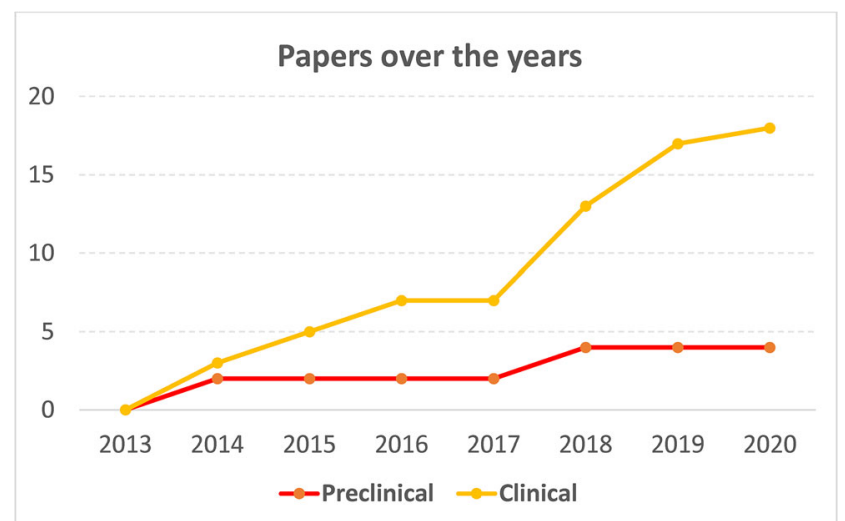

Fig. 2 Cumulative number of preclinical and clinical studies on BMAC injective OA treatment
(PRP), and cultured BMSCs. Knee OA was induced by anterior cruciate ligament transection in all four studies, also associated with meniscectomy in two studies. Bone marrow was harvested from the iliac crest in three studies and from the proximal tibia in one study. The used animals included rabbit (two studies), sheep, and goat (one study each), which were sacrificed from six to 20 weeks after the intra-articular injections, and then evaluated through macroscopic and radiologic evaluation, histological examination, and immunohistochemical analysis.

The macroscopic evaluation of articular cartilage was described in three studies, all showing better results in animals treated with BMAC compared with control groups. Song et al. [29] and Singh et al. [30] reported less severe cartilage deterioration compared with saline. In addition, beside showing the benefits vs. saline, Wang et al. [28] documented in the BMAC group only focal and superficial cartilage erosion and mild osteophyte development with respect to the PRP group, where obvious extensive and full-thickness cartilage defects combined with marked osteophyte development were reported. Singh et al. [30] performed also a radiological evaluation, confirming the positive results by showing less severe signs of OA (including osteophyte formation, subchondral bone sclerosis, and articular surface irregularity) when isolated non-cultured BMSCs were injected compared with saline. 


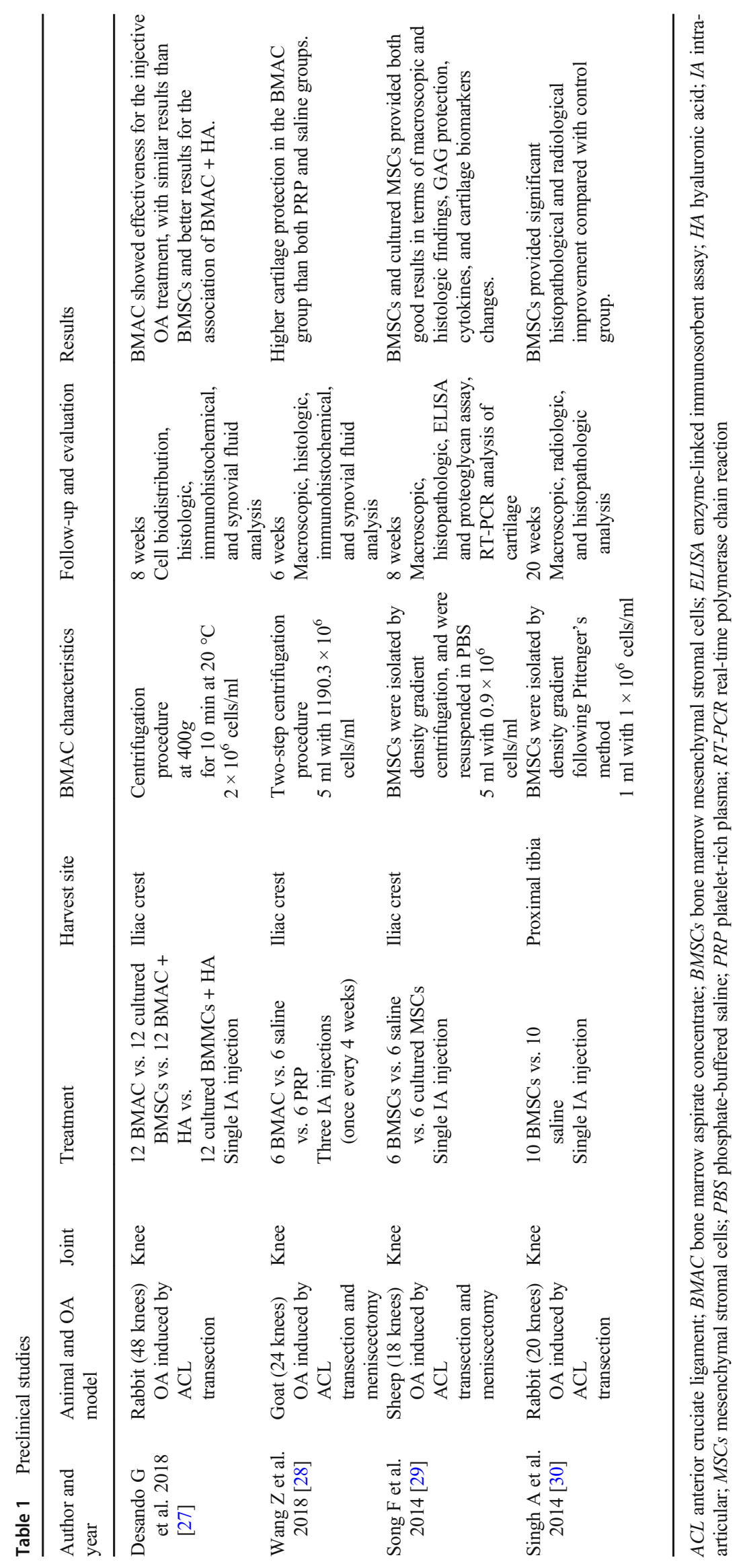


The histological analysis was performed in all studies, reporting better results in the BMAC group, with degenerative changes and articular cartilage erosion limited within the superficial layer, normal chondrocyte density, and abundant extracellular matrix preserved in the lower layers. Interestingly Desando et al. [27] in a rabbit model demonstrated similar results with respect to the use of cultured BMSCs and better histological findings in joints treated with the combination of BMAC and HA with respect to BMAC alone. Moreover, they underlined the cell homing pattern: MSCs preferentially migrated toward tissue areas showing OA features in the meniscus and cartilage, and near inflammatory zones in the synovial membrane, and the combination with HA contributed to boost cell migration toward articular cartilage. Desando et al. [27] also investigated immunohistochemical characteristics, showing a downregulation of type I collagen and TNF- $\alpha$ in articular cartilage after BMAC treatment. Finally, Song et al. [29] evaluated GAG content showing an increase in the proteoglycan concentration in the cellular treatment groups. Moreover, they analyzed the gene expression of Col2A1, Aggrecan, and MMP-13 underlining a significant increase in Col2A1 and Aggrecan, and a decrease in MMP-13 in both non-cultured BMSC and cultured MSC groups compared with the saline group.

\section{Clinical studies}

Out of the 18 clinical articles found (Table 2), six were retrospective case series, five were retrospective comparative studies, four were randomized controlled trials (RCTs), and three were prospective case series. Eleven studies focused on knee OA [31-41], two focused on hip OA [42, 43], two focused on shoulder OA [44, 45], while the other three studies described several joints affected by OA [46-48]. Intra-articular BMAC injections were performed in 17 studies, while in one study [36], the injection was performed within the tibial and femoral subchondral bone of the knee. The most common injection schedule was the single injection (16 articles), while a four injection schedule was studied in two articles [37, 42]. In 11 studies, BMAC was the only product injected, while in the other seven studies, other products were combined including PRP, platelet lysate, or minimally processed adipose tissue. In the nine studies with control groups, the effectiveness of BMAC was compared with other injective treatments such as PRP, micro-fragmented adipose tissue, whole bone marrow, cultured MSCs, placebo, or other treatments such as exercise and total knee arthroplasty. The BMAC harvest site was the anterior-superior (ASIS) or postero-superior iliac spine (PSIS) (14 PSIS, 3 ASIS, and 1 ASIS or PSIS). The total number of subjects included and treated with BMAC injections was 4626 , and the mean trial duration was 20 months, ranging from two months to 12 years of follow-up, with all but one study [36] lasting less than 2.5 years. The evaluation with the modified Coleman Methodology Score showed an overall poor methodology of the included studies, with an average score of 36.6 points out of 100 (range 23-60), as reported in Table 2.

The main finding of the included studies was an overall improvement in pain and function in OA patients treated with BMAC injections, with similar results obtained for the different joints evaluated. However, the comparative studies were not able to prove superiority over the other intra-articular options and, in the only placebo-blinded RCT [33], BMAC did not show superiority over saline at 12 months of follow-up. On the other hand, intraarticular BMAC injections (combined with platelet products) demonstrated better results than exercise therapy in knee OA patients at 24 months of follow-up [35], and subchondral BMAC injections reported similar results compared with total knee arthroplasty in younger patients with knee OA secondary to corticosteroid-related osteonecrosis at an average of 12 years of follow-up [36].

Shaw et al. [37] underlined the importance of the injection schedule, suggesting that multiple BMAC injections can be more effective than a single injection, and reported additional benefit with each subsequent treatment both for knee and hip OA. In addition, Centeno et al. [39] suggested that patients receiving a BMAC injection with a higher concentration of cells reported a better pain outcome in comparison with the lower dose group. The role of OA severity was underlined in two studies [41, 43] showing better clinical results after intraarticular BMAC injections in patients with moderate OA compared with those with severe OA (Kellgren-Lawrence grade 4). Finally, the possibility to improve the results by combining different products was investigated in two comparative studies $[40,47]$, where the combination of BMAC and adipose tissue did not prove superiority with respect to BMAC alone.

Safety was documented by all studies, with no severe adverse events related to the injective procedures. The most common reported adverse effects were temporary pain or joint swelling during the first weeks after BMAC injection, followed by grinding, popping, and snapping sensations with specific movements. Centeno et al. [47] documented in a multi-center safety analysis a $12 \%$ rate of adverse events in a large group of OA patients treated with intraarticular injections of BMAC. In particular, patients receiving BMAC injections alone reported fewer adverse events with respect to patients treated with intra-articular combined injections (e.g., BMAC plus adipose tissue). The same group also reported in another study [35] one patient with a persistent popliteal fossa fluid accumulation after the injection procedure, which was aspirated and resolved. Finally, Hernigou et al. [36] reported a higher safety rate in patients treated with knee subchondral BMAC injections compared with patients who underwent total knee arthroplasty. 


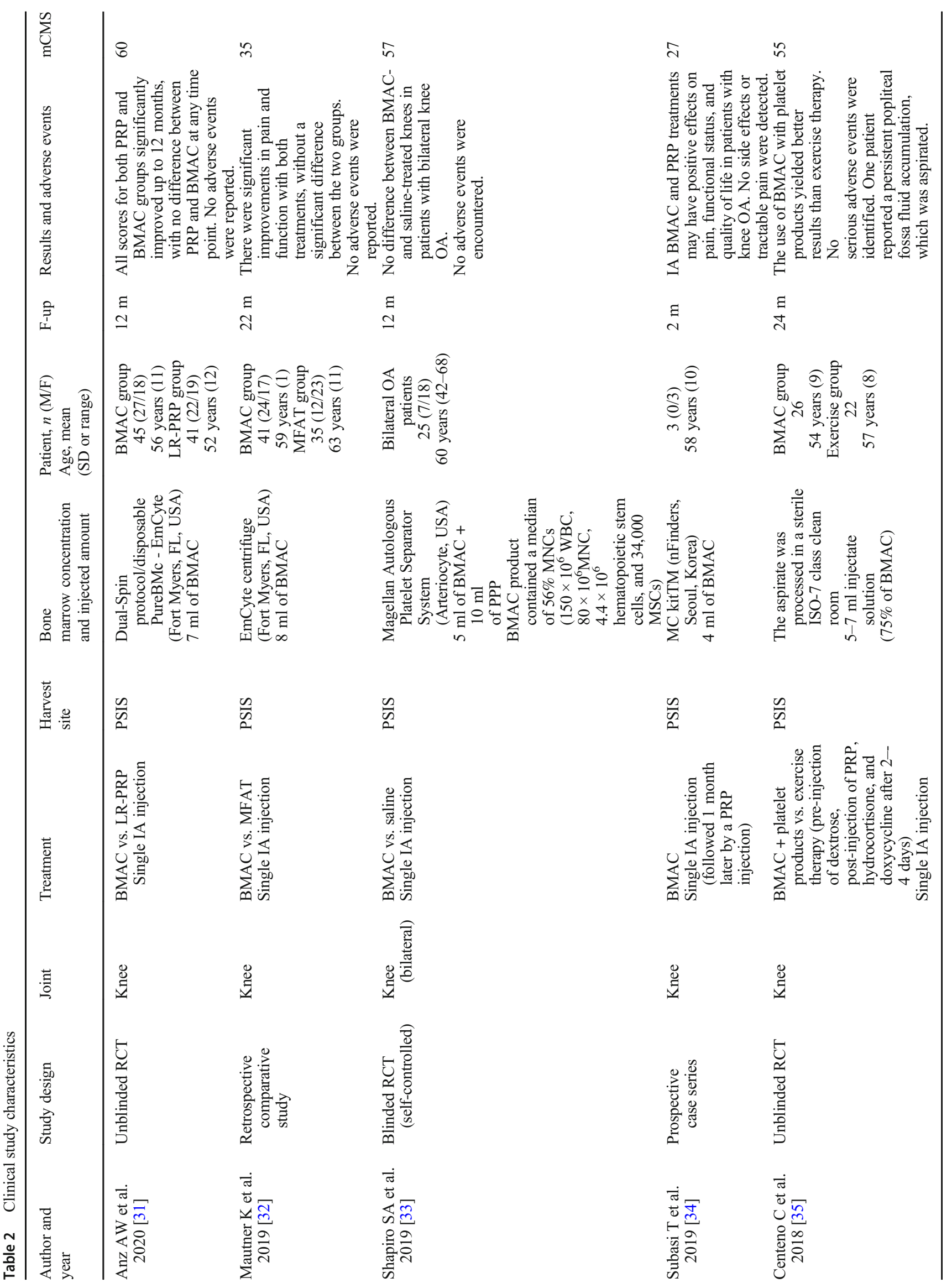




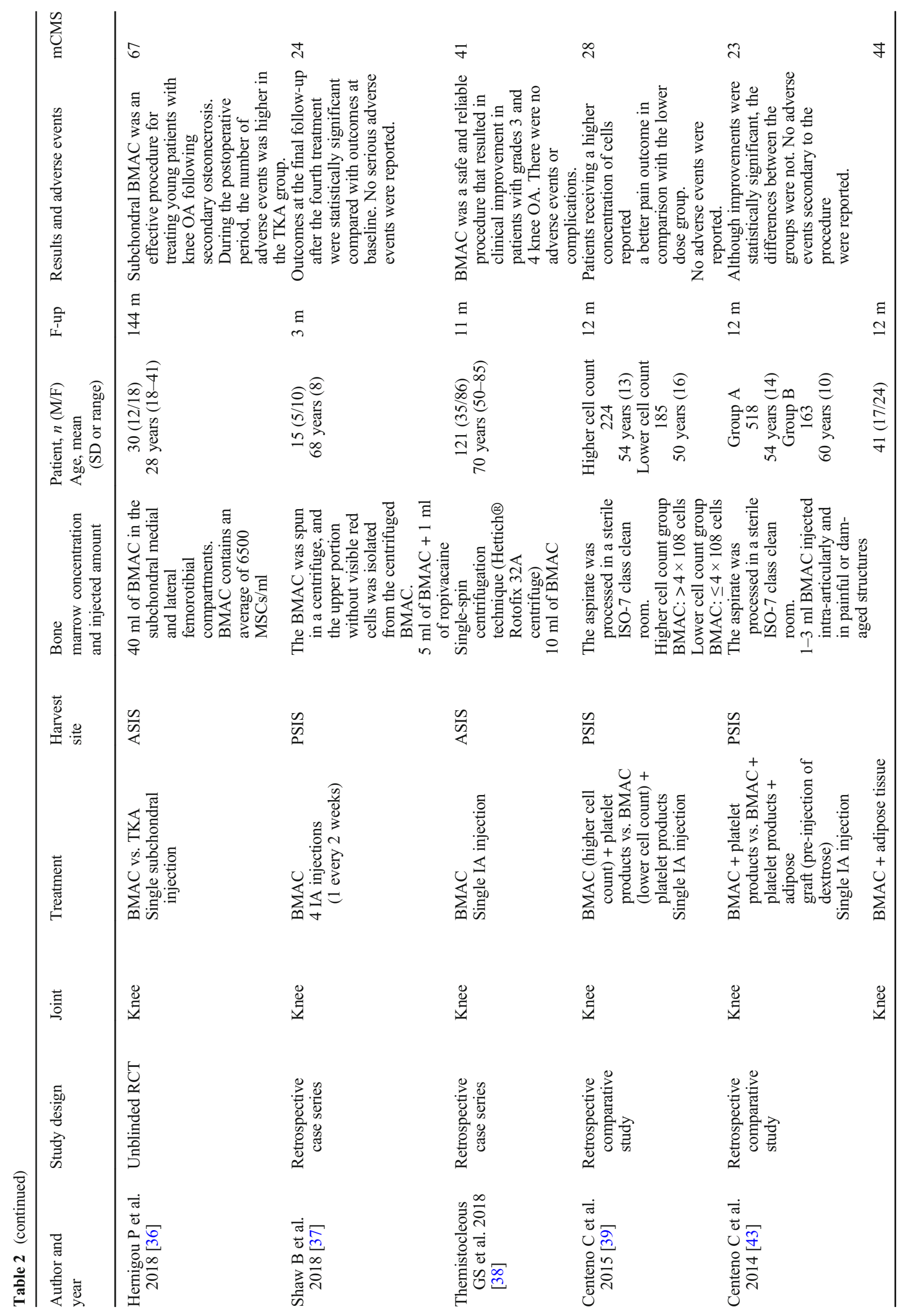




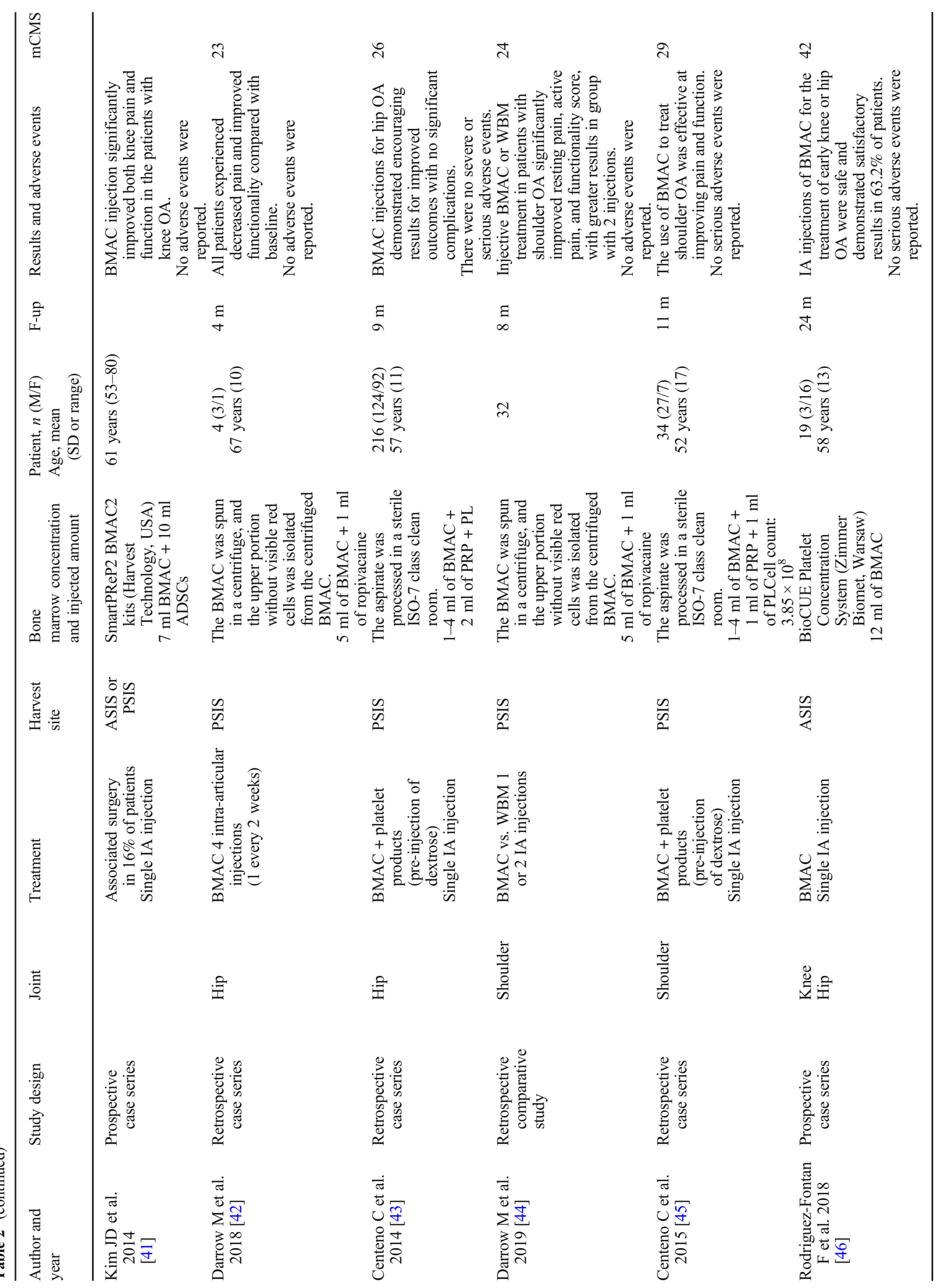




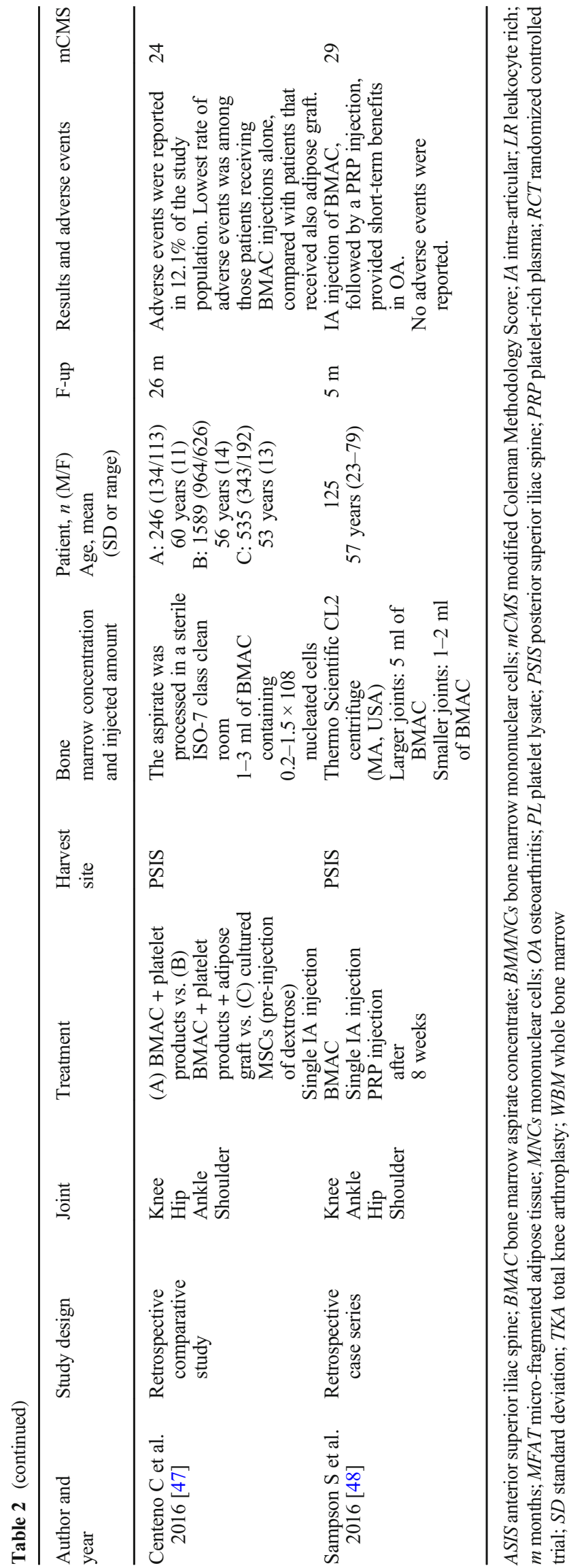

\section{Discussion}

The main finding of this systematic review is that the available preclinical and clinical studies suggest safety and overall positive results of the injective treatment with BMAC in joints affected by OA. This research highlighted a growing number of clinical trials published in the last years. Nevertheless, the literature analysis also underlined the limits of this young field, with only a few preclinical studies supporting the rationale of BMAC injections, as well as an overall low quality of evidence of the clinical studies.

BMAC is increasingly used as injective treatment of OA, with the rationale relying on the transplantation of the entire bone marrow niche which contains MSCs, haematopoietic precursors, monocytes, and endothelial cells, as well as a great array of soluble factors $[14,49,50]$. BMSCs have the capacity to differentiate toward several lineages (i.e., chondrocytes, osteoblast, adipocytes) and to produce soluble factors, which may positively affect the joint homeostasis and eventually contribute to relief pain and to improve joint function [21, $51,52]$. In particular, MSCs possess immunomodulatory, anti-inflammatory, anti-apoptotic, proliferative, and chemoattractive functions, and can coordinate the differentiation process of functional tissue regeneration in host cells [53]. However, the amount of BMSCs in BMA is not elevated, with a study investigating $\mathrm{CD} 34^{-}, \mathrm{CD} 45^{\text {low }}$, and $\mathrm{CD} 271^{\text {high }}$ positive cells underlying a low $0.04 \%$ rate [54]. Quantifying BMSCs has been historically based on the number of colony-forming units (CFU) that emerge from in vitro culture of BMA samples, with studies estimating between 109 and 664 CFU per milliliter of BMA [55]. BMAC only slightly increases the concentration of BMSCs, leading to a much lower number of BMSCs compared with what was administered in studies on the injection of expanded BMSCs, where a range from $5 \times 10^{6} \mathrm{cell} / \mathrm{ml}$ to $24 \times 10^{6} \mathrm{cell} / \mathrm{ml}$ [14] has been reported. Nevertheless, compared with cultured BMSCs, BMAC contains a high number of platelets rich in growth factors, cytokines, and chemokines, including transforming growth factor-beta (TGF- $\beta$ ), interleukin-1 receptor antagonist (IL-1ra), platelet-derived growth factor (PDGF), bone morphogenetic protein (BMP)-2 and -7, and vascular endothelial growth factor (VEGF). These growth factors are involved in several pathways crucial for cell maintenance and function, for differentiation, for extracellular matrix production, and for the regulation of cell catabolic/anabolic activity [56-58]. Accordingly, this combination of cells and bioactive proteins makes BMAC a unique product among the orthobiologics presently available, and may present the potential to alter the disease course and not just to decrease pain [59]. Beside this rationale, the increasing use of BMAC is also due to the severe restrictions and regulatory issues related to other strategies to exploit MSCs, which involve extended cell manipulation and in vitro cultivation. This, together with the risk of infection, 
contamination, or cell trans-differentiation [17-19], makes cultured MSCs use extremely limited in the clinical practice both in Europe and in the USA [15]. On the other hand, the use of BMAC is authorized by the US Food and Drug Administration (FDA) because it is considered "minimally manipulated," although its use is foreseen only for a homologous purpose and without involving a combination of cells or tissues with another substance [15]. According to this regulatory window, several different products have been proposed in the clinical practice to produce BMAC.

BMAC is frequently obtained through density gradient centrifugation of BMA, usually collected from the posterior or anterior iliac crest, and rarely from the distal femoral or proximal tibial metaphysis, the latter option being proposed in particular for knee pathology treatment [60]. The harvest site may play an important role for the product obtained. In a recent study, Davies et al. [61] suggested that the pelvis was superior to femur and tibia in terms of the number of stem cells isolated, even though there was no significant difference in the phenotype of the cells isolated from different locations. Moreover, Pierini et al. [62] demonstrated a higher quality of BMA when it was collected from the posterior rather than anterior iliac crest in terms of number of colony-founding connective-tissue progenitors. Beside the anatomical site, other factors can influence the quality of BMAC. Some authors reported a variable stem cell quantity and quality depending on age, including an age-related reduction in the absolute number of MSCs within BMA and a decreased proliferative and differentiative capacity [63, 64]. Finally, the production process itself might influence the biological properties of BMAC. The collected BMA is usually centrifuged directly in the operating room, and nucleated cells (stem cells, monocytes, lymphocytes) and proteins (growth factors, cytokines, and chemokines) are concentrated by removing most of the red cells and plasma. Then, BMA is reduced in volume with a cell separator to obtain 3-8 ml of BMAC, and the final volume used will depend from the clinical application. Even though these steps are common to most of the procedures developed to obtain BMAC by BMA centrifugation, there are several commercial systems suitable for producing BMAC. The lack of standardization of the producing techniques (such as differences in BMA starting volumes, centrifuge devices, and many other methodological differences) leads to several biological products with different progenitor cells and platelet number, other than different concentrations of growth factors and cytokines $[65,66]$. This aspect may hinder the possibility to compare literature results and to understand the real potential of BMAC as OA injective treatment.

Despite the many still controversial aspects, BMAC is technically easy and presents the advantages of overcoming the need for culture expansion, thus reducing the risk of infection and avoiding the risk of allogeneic diseases [67]. Thus, BMAC use is growing exponentially in the clinical practice for several orthopaedic procedures, including the treatment for OA $[68,69]$. In a previous literature review, Di Matteo et al. underlined that currently BMAC, together with adipose tissue-derived concentrates, were the most common strategies to exploit MSC potential through minimal manipulation, both showing promising results for knee OA, but also an overall poor methodology [65]. Nevertheless, it is important to underline that, while a large literature is available for other types of BMAC applications, the clinical OA application is poorly justified by preclinical evidence on this specific treatment indication. Only few preclinical studies focused on the intraarticular injective use of BMAC for OA, while the majority of these studies focused on culture-expanded BMSCs or on surgical applications of BMAC, including the treatment of focal cartilage defects (surgical augmentation or scaffoldbased applications) [14, 70, 71]. The few preclinical studies in the literature suggested that BMAC can affect OA progression in the animal models, but they also underlined its limited potential, and the possibility to further exploit it through different production protocols as well as the combination of injective carriers to positively affect cell migration and favor longer-lasting homeostatic and disease-modifying effects $[51,72]$.

BMAC has been studied mainly for the knee injective treatment, while only few studies focused on shoulder, hip, or ankle OA. Overall, most of these studies converged on the safety and benefit of BMAC for OA symptom management. Better results were found for BMAC injections against exercise therapy in 48 patients with symptomatic knee OA, where this injective treatment suggested a greater impact on patients' knee function [35]. Moreover, BMAC injections were found to provide clinical results comparable with TKA in a population of 30 young patients affected by osteonecrosis-related OA but with lower adverse events, thus suggesting, with all the limits inherent of a small survey, the possibility to delay joint replacement through the application of a regenerative therapy [36]. However, it is important to underline that no comparative study reported a superiority of BMAC against other injective treatments and, more importantly, the only available placebo-controlled RCT was not able to show any difference between BMAC- and saline-treated knees in patients with bilateral knee OA. Injective treatments, especially the "regenerative" ones, present a high and long-lasting placebo effect [8], and high-level trials will have to prove that BMAC can exceed the mere placebo effect, to confirm the promising findings suggested by the published studies and justify its use in the clinical practice.

A new application has been recently proposed to further exploit the potential of BMAC by targeting the subchondral bone which is commonly affected by the OA processes [36]. This strategy is supported by histological studies underlying different potential patterns of $\mathrm{OA}$ initiation, including initial subchondral bone alterations which may progress to the 
destruction of the overlying articular surface [73, 74]. Accordingly, the subchondral bone is becoming more and more a target and subchondral injective therapies are gaining an increasing interest, ranging from bone substitutes to biological treatments [75]. In particular, some authors reported the safety and effectiveness of subchondral PRP injections for the treatment of severe knee and hip OA [76-78]. Sanchez et al. [79] suggested a superior clinical outcome at six and 12 months for the combination of subchondral and intraarticular PRP injections when compared with intra-articular injections alone. The same authors suggested the possibility of subchondral PRP injections to delay arthroplasty in severe knee OA [80]. Similarly, Vad et al. [81] showed an improvement in pain and function in patients with knee OA treated with subchondral injections of BMA as an alternative to total knee replacement when other minimally invasive therapies have failed. Still, the subchondral application of BMAC has currently a limited scientific support and, as per intra-articular injections, more high-level studies are needed to understand if this could represent a valid option among biological treatment strategies for OA.

This systematic review presents several limitations reflecting the limitations of the available studies. In fact, the overall level of evidence was low; only four RCTs with a small size are available, while the other studies were retrospective comparative studies or case series. Another significant aspect is the absence of a proper control group comparison, with only one placebo-controlled trial and overall few and heterogeneous comparative studies performed. Many differences were documented among the included studies in terms of not standardized treatment protocols and different outcome measures. To this regard, many authors evaluated BMAC used with other orthobiologics, such as PRP or adipose tissue, hindering the possibility to isolate and assess the efficacy of BMAC as monotherapy. Moreover, BMAC preparation method, characteristics, and application modalities (MSC count, injected amount, injection schedule) were often different or not even reported by the authors. In addition, most of the studies used different scoring systems at different follow-ups, making it difficult to compare and merge results, thus impairing the possibility to perform a meta-analysis and to draw clear conclusions. Finally, the relatively short followup in most of these studies leaves concerns regarding the durability of the treatment results with BMAC for OA. Albeit being considered minimally invasive, this treatment still requires a surgical approach and results should prove significant and long-lasting to justify its use vs. less invasive homeostatic treatments [69].

In conclusion, this systematic review suggested a growing interest in the field of BMAC injections for the treatment of $\mathrm{OA}$, with promising results in preclinical and clinical studies in terms of safety and effectiveness. Nevertheless, the current knowledge is still preliminary, the literature includes scarce preclinical evidence supporting BMAC rationale for this application, and clinical studies present significant heterogeneity, few patients, short-term follow-up, and overall poor methodology. Many aspects remain to be clarified to optimize the potential of BMAC, including methods of harvest, centrifugation, timing of injections, and application method, and to provide a standardized method targeted for OA treatment. Preclinical research is still needed to optimize BMAC use, as well as high-level large controlled trials to better understand the real potential of BMAC injections for the treatment of patients affected by OA.

Code availability Not applicable.

Funding information Open access funding provided by Alma Mater Studiorum - Università di Bologna within the CRUI-CARE Agreement. This study was supported by the project Giovane Ricercatore GR-201602361990 funded by the Italian Ministry of Health.

Data availability Not applicable.

\section{Compliance with ethical standards}

Conflict of interest Stefano Zaffagnini has received institutional support from Fidia Farmaceutici, Cartiheal, IGEA Clinical Biophysics, Biomet, and Kensey Nash; grant support from I+; and royalties from Springer.

The other authors declare no conflict of interest.

Ethics approval Not applicable.

Consent to participate Not applicable.

Consent for publication Not applicable.

Open Access This article is licensed under a Creative Commons Attribution 4.0 International License, which permits use, sharing, adaptation, distribution and reproduction in any medium or format, as long as you give appropriate credit to the original author(s) and the source, provide a link to the Creative Commons licence, and indicate if changes were made. The images or other third party material in this article are included in the article's Creative Commons licence, unless indicated otherwise in a credit line to the material. If material is not included in the article's Creative Commons licence and your intended use is not permitted by statutory regulation or exceeds the permitted use, you will need to obtain permission directly from the copyright holder. To view a copy of this licence, visit http://creativecommons.org/licenses/by/4.0/.

\section{References}

1. Mobasheri A, Batt M (2016) An update on the pathophysiology of osteoarthritis. Ann Phys Rehabil Med 59:333-339. https://doi.org/ 10.1016/j.rehab.2016.07.004

2. Wallace IJ, Worthington S, Felson DT et al (2017) Knee osteoarthritis has doubled in prevalence since the mid-20th century. Proc Natl Acad Sci U S A 114:9332-9336. https://doi.org/10.1073/pnas. 1703856114 
3. Zhang Y, Jordan JM (2010) Epidemiology of osteoarthritis. Clin Geriatr Med 26:355-369. https://doi.org/10.1016/j.cger.2010.03. 001

4. Neogi T, Zhang Y (2013) Epidemiology of osteoarthritis. Rheum Dis Clin N Am 39:1-19. https://doi.org/10.1016/j.rdc.2012.10.004

5. Goldring SR, Goldring MB (2006) Clinical aspects, pathology and pathophysiology of osteoarthritis. J Musculoskelet Neuronal Interact 6:376-378

6. Filardo G, Kon E, Longo UG et al (2016) Non-surgical treatments for the management of early osteoarthritis. Knee Surg Sports Traumatol Arthrosc 24:1775-1785. https://doi.org/10.1007/ s00167-016-4089-y

7. Losina E, Paltiel AD, Weinstein AM et al (2015) Lifetime medical costs of knee osteoarthritis management in the United States: impact of extending indications for total knee arthroplasty. Arthritis Care Res (Hoboken) 67:203-215. https://doi.org/10.1002/acr. 22412

8. Previtali D, Merli G, Di Laura FG, Candrian C, Zaffagnini S, Filardo G (2020) The long-lasting effects of "placebo injections" in knee osteoarthritis: a meta-analysis. Cartilage: 1947603520906597. https://doi.org/10.1177/1947603520906597

9. Filardo G, Previtali D, Napoli F, Candrian C, Zaffagnini S, Grassi A (2020) PRP injections for the treatment of knee osteoarthritis: a meta-analysis of randomized controlled trials. Cartilage: 1947603520931170. https://doi.org/10.1177/1947603520931170

10. Caplan AI (2005) Review: mesenchymal stem cells: cell-based reconstructive therapy in orthopedics. Tissue Eng 11:1198-1211. https://doi.org/10.1089/ten.2005.11.1198

11. Chen FH, Tuan RS (2008) Mesenchymal stem cells in arthritic diseases. Arthritis Res Ther 10:223. https://doi.org/10.1186/ar2514

12. Kuci S, Henschler R, Muller I, Biagi E, Meisel R (2012) Basic biology and clinical application of multipotent mesenchymal stromal cells: from bench to bedside. Stem Cells Int 2012:185943. https://doi.org/10.1155/2012/185943

13. Friedenstein AJ, Piatetzky S II, Petrakova KV (1966) Osteogenesis in transplants of bone marrow cells. J Embryol Exp Morphol 16: 381-390

14. Filardo G, Madry H, Jelic M, Roffi A, Cucchiarini M, Kon E (2013) Mesenchymal stem cells for the treatment of cartilage lesions: from preclinical findings to clinical application in orthopaedics. Knee Surg Sports Traumatol Arthrosc 21:1717-1729. https://doi.org/10. 1007/s00167-012-2329-3

15. Administration FaD (2017) Regulatory considerations for human cells, tissues, and cellular and tissuebased products: minimal manipulation and homologous use

16. Pagani S, Borsari V, Veronesi F et al (2017) Increased chondrogenic potential of mesenchymal cells from adipose tissue versus bone marrow-derived cells in osteoarthritic in vitro models. J Cell Physiol 232:1478-1488. https://doi.org/10.1002/jcp.25651

17. Turner L, Knoepfler P (2016) Selling stem cells in the USA: assessing the direct-to-consumer industry. Cell Stem Cell 19:154 157. https://doi.org/10.1016/j.stem.2016.06.007

18. Song L, Tuan RS (2004) Transdifferentiation potential of human mesenchymal stem cells derived from bone marrow. FASEB J 18: 980-982. https://doi.org/10.1096/fj.03-1100fje

19. Herberts CA, Kwa MS, Hermsen HP (2011) Risk factors in the development of stem cell therapy. J Transl Med 9:29. https://doi. org/10.1186/1479-5876-9-29

20. Imam MA, Mahmoud SSS, Holton J, Abouelmaati D, Elsherbini Y, Snow M (2017) A systematic review of the concept and clinical applications of bone marrow aspirate concentrate in orthopaedics. SICOT J 3:17. https://doi.org/10.1051/sicotj/2017007

21. Filardo G, Perdisa F, Roffi A, Marcacci M, Kon E (2016) Stem cells in articular cartilage regeneration. J Orthop Surg Res 11:42. https:// doi.org/10.1186/s13018-016-0378-x
22. Bannuru RR, Osani MC, Vaysbrot EE et al (2019) OARSI guidelines for the non-surgical management of knee, hip, and polyarticular osteoarthritis. Osteoarthr Cartil 27:1578-1589. https://doi.org/10.1016/j.joca.2019.06.011

23. Liberati A, Altman DG, Tetzlaff J et al (2009) The PRISMA statement for reporting systematic reviews and meta-analyses of studies that evaluate health care interventions: explanation and elaboration. PLoS Med 6:e1000100. https://doi.org/10.1371/journal.pmed. 1000100

24. Kon E, Verdonk P, Condello V et al (2009) Matrix-assisted autologous chondrocyte transplantation for the repair of cartilage defects of the knee: systematic clinical data review and study quality analysis. Am J Sports Med 37(Suppl 1):156S-166S. https://doi.org/10. $1177 / 0363546509351649$

25. Malanga GA, Dona S, Borg-Stein J, Auriemma M, Singh JR (2018) Refractory knee osteoarthritis: adipose-derived stromal cells versus bone marrow aspiration concentrate. PM R 10:524-532. https://doi. org/10.1016/j.pmrj.2018.03.021

26. Shapiro SA, Kazmerchak SE, Heckman MG, Zubair AC, O'Connor MI (2017) A prospective, single-blind, placebocontrolled trial of bone marrow aspirate concentrate for knee osteoarthritis. Am J Sports Med 45:82-90. https://doi.org/10.1177/ 0363546516662455

27. Desando G, Bartolotti I, Cavallo C et al (2018) Short-term homing of hyaluronan-primed cells: therapeutic implications for osteoarthritis treatment. Tissue Eng Part C Methods 24:121-133. https://doi. org/10.1089/ten.TEC.2017.0336

28. Wang Z, Zhai C, Fei H et al (2018) Intraarticular injection autologous platelet-rich plasma and bone marrow concentrate in a goat osteoarthritis model. J Orthop Res. https://doi.org/10.1002/jor. 23877

29. Song F, Tang J, Geng R et al (2014) Comparison of the efficacy of bone marrow mononuclear cells and bone mesenchymal stem cells in the treatment of osteoarthritis in a sheep model. Int J Clin Exp Pathol 7:1415-1426

30. Singh A, Goel SC, Gupta KK et al (2014) The role of stem cells in osteoarthritis: an experimental study in rabbits. Bone Joint Res 3: 32-37. https://doi.org/10.1302/2046-3758.32.2000187

31. Anz AW, Hubbard R, Rendos NK, Everts PA, Andrews JR, Hackel JG (2020) Bone marrow aspirate concentrate is equivalent to platelet-rich plasma for the treatment of knee osteoarthritis at 1 year: a prospective, randomized trial. Orthop J Sports Med 8: 2325967119900958. https://doi.org/10.1177/2325967119900958

32. Mautner K, Bowers R, Easley K, Fausel Z, Robinson R (2019) Functional outcomes following microfragmented adipose tissue versus bone marrow aspirate concentrate injections for symptomatic knee osteoarthritis. Stem Cells Transl Med 8:1149-1156. https:// doi.org/10.1002/sctm.18-0285

33. Shapiro SA, Arthurs JR, Heckman MG et al (2019) Quantitative T2 MRI mapping and 12-month follow-up in a randomized, blinded, placebo controlled trial of bone marrow aspiration and concentration for osteoarthritis of the knees. Cartilage 10:432-443. https:// doi.org/10.1177/1947603518796142

34. Subasi V, Ekiz T (2019) Bone marrow aspiration concentrate and platelet-rich plasma in the treatment of knee osteoarthritis: a report of three cases. Complement Ther Clin Pract 34:113-115. https:// doi.org/10.1016/j.ctcp.2018.11.005

35. Centeno C, Sheinkop M, Dodson E et al (2018) A specific protocol of autologous bone marrow concentrate and platelet products versus exercise therapy for symptomatic knee osteoarthritis: a randomized controlled trial with 2 year follow-up. J Transl Med 16:355. https://doi.org/10.1186/s12967-018-1736-8

36. Hernigou P, Auregan JC, Dubory A, Flouzat-Lachaniette $\mathrm{CH}$, Chevallier N, Rouard H (2018) Subchondral stem cell therapy versus contralateral total knee arthroplasty for osteoarthritis following 
secondary osteonecrosis of the knee. Int Orthop 42:2563-2571. https://doi.org/10.1007/s00264-018-3916-9

37. Shaw B, Darrow M, Derian A (2018) Short-term outcomes in treatment of knee osteoarthritis with 4 bone marrow concentrate injections. Clin Med Insights Arthritis Musculoskelet Disord 11: 1179544118781080. https://doi.org/10.1177/1179544118781080

38. Themistocleous GS, Chloros GD, Kyrantzoulis IM et al (2018) Effectiveness of a single intra-articular bone marrow aspirate concentrate (BMAC) injection in patients with grade 3 and 4 knee osteoarthritis. Heliyon 4:e00871. https://doi.org/10.1016/j.heliyon. 2018.e00871

39. Centeno CJ, Al-Sayegh H, Bashir J, Goodyear S, Freeman MD (2015) A dose response analysis of a specific bone marrow concentrate treatment protocol for knee osteoarthritis. BMC Musculoskelet Disord 16:258. https://doi.org/10.1186/s12891-015-0714-z

40. Centeno C, Pitts J, Al-Sayegh H, Freeman M (2014) Efficacy of autologous bone marrow concentrate for knee osteoarthritis with and without adipose graft. Biomed Res Int 2014:370621. https:// doi.org/10.1155/2014/370621

41. Kim JD, Lee GW, Jung GH et al (2014) Clinical outcome of autologous bone marrow aspirates concentrate (BMAC) injection in degenerative arthritis of the knee. Eur J Orthop Surg Traumatol 24: 1505-1511. https://doi.org/10.1007/s00590-013-1393-9

42. Darrow M, Shaw B, Darrow B, Wisz S (2018) Short-term outcomes of treatment of hip osteoarthritis with 4 bone marrow concentrate injections: a case series. Clin Med Insights Case Rep 11: 1179547618791574. https://doi.org/10.1177/1179547618791574

43. Centeno CPJ, Al-Sayegh H, Freeman MD (2014) Efficacy and safety of bone marrow concentrate for osteoarthritis of the hip; treatment registry results for 196 patients. J Stem Cell Res Ther 4: 7. https://doi.org/10.4172/2157-7633.1000242

44. Darrow MSB, Schmidt N, Boeger G, Budgett S (2019) Treatment of shoulder osteoarthritis and rotator cuff tears with bone marrow concentrate and whole bone marrow injections. Cogent Med Vol 6. https://doi.org/10.1080/2331205X.2019.1628883

45. Centeno CJ, Al-Sayegh H, Bashir J, Goodyear S, Freeman MD (2015) A prospective multi-site registry study of a specific protocol of autologous bone marrow concentrate for the treatment of shoulder rotator cuff tears and osteoarthritis. J Pain Res 8:269-276. https://doi.org/10.2147/JPR.S80872

46. Rodriguez-Fontan F, Piuzzi NS, Kraeutler MJ, Pascual-Garrido C (2018) Early clinical outcomes of intra-articular injections of bone marrow aspirate concentrate for the treatment of early osteoarthritis of the hip and knee: a cohort study. PM R 10:1353-1359. https:// doi.org/10.1016/j.pmrj.2018.05.016

47. Centeno CJ, Al-Sayegh H, Freeman MD, Smith J, Murrell WD, Bubnov R (2016) A multi-center analysis of adverse events among two thousand, three hundred and seventy two adult patients undergoing adult autologous stem cell therapy for orthopaedic conditions. Int Orthop 40:1755-1765. https://doi.org/10.1007/s00264016-3162-y

48. Sampson S, Smith J, Vincent H, Aufiero D, Zall M, Botto-vanBemden A (2016) Intra-articular bone marrow concentrate injection protocol: short-term efficacy in osteoarthritis. Regen Med 11:511520. https://doi.org/10.2217/rme-2016-0081

49. Fortier LA, Potter HG, Rickey EJ et al (2010) Concentrated bone marrow aspirate improves full-thickness cartilage repair compared with microfracture in the equine model. J Bone Joint Surg Am 92: 1927-1937. https://doi.org/10.2106/JBJS.I.01284

50. Johnson RG (2014) Bone marrow concentrate with allograft equivalent to autograft in lumbar fusions. Spine (Phila Pa 1976) 39:695700. https://doi.org/10.1097/BRS.0000000000000254

51. Roffi A, Nakamura N, Sanchez M, Cucchiarini M, Filardo G (2018) Injectable systems for intra-articular delivery of mesenchymal stromal cells for cartilage treatment: a systematic review of preclinical and clinical evidence. Int J Mol Sci 19. https://doi.org/10.3390/ ijms 19113322

52. Lopa S, Colombini A, Moretti M, de Girolamo L (2019) Injective mesenchymal stem cell-based treatments for knee osteoarthritis: from mechanisms of action to current clinical evidences. Knee Surg Sports Traumatol Arthrosc 27:2003-2020. https://doi.org/10. 1007/s00167-018-5118-9

53. Gaul F, Bugbee WD, Hoenecke HR Jr, D’Lima DD (2019) A review of commercially available point-of-care devices to concentrate bone marrow for the treatment of osteoarthritis and focal cartilage lesions. Cartilage 10:387-394. https://doi.org/10.1177/ 1947603518768080

54. de Girolamo L, Bertolini G, Cervellin M, Sozzi G, Volpi P (2010) Treatment of chondral defects of the knee with one step matrixassisted technique enhanced by autologous concentrated bone marrow: in vitro characterisation of mesenchymal stem cells from iliac crest and subchondral bone. Injury 41:1172-1177. https://oi.org/ 10.1016/j.injury.2010.09.027

55. Anz AW, Hackel JG, Nilssen EC, Andrews JR (2014) Application of biologics in the treatment of the rotator cuff, meniscus, cartilage, and osteoarthritis. J Am Acad Orthop Surg 22:68-79. https://doi. org/10.5435/JAAOS-22-02-68

56. Miyazawa K, Shinozaki M, Hara T, Furuya T, Miyazono K (2002) Two major Smad pathways in TGF-beta superfamily signalling. Genes Cells 7:1191-1204. https://doi.org/10.1046/j.1365-2443. 2002.00599.x

57. Mariani E, Pulsatelli L, Facchini A (2014) Signaling pathways in cartilage repair. Int J Mol Sci 15:8667-8698. https://doi.org/10. 3390/ijms 15058667

58. Fortier LA, Barker JU, Strauss EJ, McCarrel TM, Cole BJ (2011) The role of growth factors in cartilage repair. Clin Orthop Relat Res 469:2706-2715. https://doi.org/10.1007/s11999-011-1857-3

59. Fortier LA, Strauss EJ, Shepard DO, Becktell L, Kennedy JG (2019) Biological effects of bone marrow concentrate in knee pathologies. J Knee Surg 32:2-8. https://doi.org/10.1055/s-00381676069

60. Shapiro SA, Arthurs JR (2017) Bone marrow aspiration for regenerative orthopedic intervention: technique with ultrasound guidance for needle placement. Regen Med 12:917-928. https://doi.org/10. 2217/rme-2017-0109

61. Davies BM, Snelling SJB, Quek L et al (2017) Identifying the optimum source of mesenchymal stem cells for use in knee surgery. J Orthop Res 35:1868-1875. https://doi.org/10.1002/jor.23501

62. Pierini M, Di Bella C, Dozza B et al (2013) The posterior iliac crest outperforms the anterior iliac crest when obtaining mesenchymal stem cells from bone marrow. J Bone Joint Surg Am 95:11011107. https://doi.org/10.2106/JBJS.L.00429

63. Stolzing A, Jones E, McGonagle D, Scutt A (2008) Age-related changes in human bone marrow-derived mesenchymal stem cells: consequences for cell therapies. Mech Ageing Dev 129:163-173. https://doi.org/10.1016/j.mad.2007.12.002

64. Baxter MA, Wynn RF, Jowitt SN, Wraith JE, Fairbairn LJ, Bellantuono I (2004) Study of telomere length reveals rapid aging of human marrow stromal cells following in vitro expansion. Stem Cells 22:675-682. https://doi.org/10.1634/stemcells.22-5-675

65. Hegde V, Shonuga O, Ellis S et al (2014) A prospective comparison of 3 approved systems for autologous bone marrow concentration demonstrated nonequivalency in progenitor cell number and concentration. J Orthop Trauma 28:591-598. https://doi.org/10.1097/ BOT.0000000000000113

66. Dragoo JL, Guzman RA (2020) Evaluation of the consistency and composition of commercially available bone marrow aspirate concentrate systems. Orthop J Sports Med 8:2325967119893634. https://doi.org/10.1177/2325967119893634

67. Chahla J, Dean CS, Moatshe G, Pascual-Garrido C, Serra Cruz R, LaPrade RF (2016) Concentrated bone marrow aspirate for the 
treatment of chondral injuries and osteoarthritis of the knee: a systematic review of outcomes. Orthop J Sports Med 4: 2325967115625481. https://doi.org/10.1177/2325967115625481

68. Andriolo L, Merli G, Tobar C, Altamura SA, Kon E, Filardo G (2018) Regenerative therapies increase survivorship of avascular necrosis of the femoral head: a systematic review and meta-analysis. Int Orthop 42:1689-1704. https://doi.org/10.1007/s00264-0183787-0

69. Di Matteo B, Vandenbulcke F, Vitale ND et al (2019) Minimally manipulated mesenchymal stem cells for the treatment of knee osteoarthritis: a systematic review of clinical evidence. Stem Cells Int 2019:1735242. https://doi.org/10.1155/2019/1735242

70. Desando G, Giavaresi G, Cavallo C et al (2016) Autologous bone marrow concentrate in a sheep model of osteoarthritis: new perspectives for cartilage and meniscus repair. Tissue Eng Part C Methods 22:608-619. https://doi.org/10.1089/ten.TEC.2016.0033

71. Cavallo C, Desando G, Columbaro M et al (2013) Chondrogenic differentiation of bone marrow concentrate grown onto a hylauronan scaffold: rationale for its use in the treatment of cartilage lesions. J Biomed Mater Res A 101:1559-1570. https://doi.org/10. 1002/jbm.a.34460

72. D'Arrigo D, Roffi A, Cucchiarini M, Moretti M, Candrian C, Filardo G (2019) Secretome and extracellular vesicles as new biological therapies for knee osteoarthritis: a systematic review. J Clin Med 8. https://doi.org/10.3390/jcm8111867

73. Mantripragada VP, Piuzzi NS, Zachos T, Obuchowski NA, Muschler GF, Midura RJ (2018) High occurrence of osteoarthritic histopathological features unaccounted for by traditional scoring systems in lateral femoral condyles from total knee arthroplasty patients with varus alignment. Acta Orthop 89:197-203. https:// doi.org/10.1080/17453674.2017.1398559

74. Mantripragada VP, Piuzzi NS, Zachos T, Obuchowski NA, Muschler GF, Midura RJ (2017) Histopathological assessment of primary osteoarthritic knees in large patient cohort reveal the possibility of several potential patterns of osteoarthritis initiation. Curr Res Transl Med 65:133-139. https://doi.org/10.1016/j.retram. 2017.09.002

75. Sundaram K, Vargas-Hernandez JS, Sanchez TR et al (2019) Are subchondral intraosseous injections effective and safe for the treatment of knee osteoarthritis? A systematic review. J Knee Surg 32: 1046-1057. https://doi.org/10.1055/s-0039-1677792

76. Lychagin A, Lipina M, Garkavi A et al (2020) Intraosseous injections of platelet rich plasma for knee bone marrow lesions treatment: one year follow-up. Int Orthop. https://doi.org/10.1007/ s00264-020-04546-5

77. Su K, Bai Y, Wang J, Zhang H, Liu H, Ma S (2018) Comparison of hyaluronic acid and PRP intra-articular injection with combined intra-articular and intraosseous PRP injections to treat patients with knee osteoarthritis. Clin Rheumatol 37:1341-1350. https://doi.org/ 10.1007/s10067-018-3985-6

78. Sanchez M, Delgado D, Sanchez P et al (2016) Combination of intra-articular and intraosseous injections of platelet rich plasma for severe knee osteoarthritis: a pilot study. Biomed Res Int 2016: 4868613. https://doi.org/10.1155/2016/4868613

79. Sanchez M, Delgado D, Pompei O et al (2019) Treating severe knee osteoarthritis with combination of intra-osseous and intra-articular infiltrations of platelet-rich plasma: an observational study. Cartilage 10:245-253. https://doi.org/10.1177/1947603518756462

80. Sanchez M, Fiz N, Guadilla J et al (2014) Intraosseous infiltration of platelet-rich plasma for severe knee osteoarthritis. Arthrosc Tech 3:e713-e717. https://doi.org/10.1016/j.eats.2014.09.006

81. V. Vad RB, E. Linnell, and J. Harrison (2016) Knee osteoarthritis treated with percutaneous chondral-bone interface optimization: a pilot trial. Surg Sci 7:1-12

Publisher's note Springer Nature remains neutral with regard to jurisdictional claims in published maps and institutional affiliations. 